\title{
Identification of novel growth phase- and media- dependent small non-coding RNAs in Streptococcus pyogenes M49 using intergenic tiling arrays
}

Nadja Patenge ${ }^{1}$, André Billion², Peter Raasch³ , Jana Normann ${ }^{1}$, Aleksandra Wisniewska-Kucper ${ }^{1}$, Julia Retey ${ }^{4}$, Valesca Boisguérin ${ }^{5}$, Thomas Hartsch ${ }^{4}$, Torsten Hain ${ }^{2}$ and Bernd Kreikemeyer ${ }^{\text {* }^{*}}$

\begin{abstract}
Background: Small non-coding RNAs (sRNAs) have attracted attention as a new class of gene regulators in both eukaryotes and bacteria. Genome-wide screening methods have been successfully applied in Gram-negative bacteria to identify sRNA regulators. Many sRNAs are well characterized, including their target mRNAs and mode of action. In comparison, little is known about sRNAs in Gram-positive pathogens. In this study, we identified novel sRNAs in the exclusively human pathogen Streptococcus pyogenes M49 (Group A Streptococcus, GAS M49), employing a whole genome intergenic tiling array approach. GAS is an important pathogen that causes diseases ranging from mild superficial infections of the skin and mucous membranes of the naso-pharynx, to severe toxic and invasive diseases.

Results: We identified 55 putative sRNAs in GAS M49 that were expressed during growth. Of these, 42 were novel. Some of the newly-identified sRNAs belonged to one of the common non-coding RNA families described in the Rfam database. Comparison of the results of our screen with the outcome of two recently published bioinformatics tools showed a low level of overlap between putative sRNA genes. Previously, 40 potential sRNAs have been reported to be expressed in a GAS M1T1 serotype, as detected by a whole genome intergenic tiling array approach. Our screen detected 12 putative sRNA genes that were expressed in both strains. Twenty sRNA candidates appeared to be regulated in a medium-dependent fashion, while eight sRNA genes were regulated throughout growth in chemically defined medium. Expression of candidate genes was verified by reverse transcriptase-qPCR. For a subset of sRNAs, the transcriptional start was determined by $5^{\prime}$ rapid amplification of CDNA ends-PCR (RACE-PCR) analysis.
\end{abstract}

Conclusions: In accord with the results of previous studies, we found little overlap between different screening methods, which underlines the fact that a comprehensive analysis of sRNAs expressed by a given organism requires the complementary use of different methods and the investigation of several environmental conditions. Despite a high conservation of sRNA genes within streptococci, the expression of sRNAs appears to be strain specific.

Keywords: Streptococcus pyogenes, Small noncoding RNAs, Virulence, Transcriptional regulation, Pathogenesis

\footnotetext{
* Correspondence: Bernd.Kreikemeyer@med.uni-rostock.de

${ }^{1}$ Institute of Medical Microbiology and Hospital Hygiene, University of

Rostock, Schillingallee 70, 18057, Rostock, Germany

Full list of author information is available at the end of the article
} 


\section{Background}

In recent years, the role of small non-coding RNAs (sRNAs) in regulation of bacterial gene expression has become more evident; however, the large number of sRNAs identified in different bacterial species was unexpected [1-3]. Even though sRNAs were conventionally regarded as inhibitory antisense regulators, a significant number of sRNAs that activate bacterial gene expression have been characterized [4]. Furthermore, regulatory mechanisms include both the stabilization and destabilization of target transcripts [5]. Bacterial sRNAs influence the expression of genes involved in processes as diverse as stress response, sugar metabolism, and surface composition [6-10]. With sRNAs representing a whole new level of post-transcriptional regulation, it is no surprise that these molecules play an important role in the tightly controlled expression of virulence factors in many pathogens $[11,12]$.

We were interested in the regulatory sRNAome of Streptococcus pyogenes (group A streptococci, GAS), a common, exclusively human pathogen that causes a variety of diseases. GAS is responsible for mild superficial infections of the skin (impetigo contagiosa) and mucosal membranes (pharyngitis and tonsillitis). Additionally, there is a high global burden of severe GAS diseases such as post-streptococcal sequelae, and severe systemic (streptococcal toxic-shock-like syndrome) or invasive infections (necrotizing fasciitis), leading to over 500,000 deaths per year $[13,14]$. The controlled expression of virulence factors plays a role in GAS infection, persistence in the host, and development of invasive diseases, which makes the investigation of virulence factors and their regulation a research priority. GAS expresses a large number of virulence factor genes coding for a variety of proteins, including surface components, lytic enzymes, proteinases, cytotoxins, superantigens, and immunoprotective proteins, that are controlled at least partially by the 30 stand-alone transcriptional regulators and 13 two-component systems identified to date in GAS [15]. Virulence factor expression in GAS is highly responsive to environmental conditions and greatly depends on the growth phase.

Little is known, however, about the importance of sRNAs for virulence-related gene regulation in GAS. An overview of small RNAs in streptococci is nicely presented by Le Rhun and Charpentier [16]. Several individual sRNAs have been identified in GAS $[17,18]$, with many more predicted by bioinformatic screens $[12,19]$. Previous analysis of sRNA expression in a GAS M1T1 serotype using an intergenic tiling array approach identified 40 potential sRNAs, with a very low predicted overlap with candidate genes [20]. The authors concluded that sRNA expression in GAS is serotypedependent. The current work focused on sRNA expression in the skin isolate GAS M49. An intergenic tiling array identified 42 novel and 13 known sRNAs. Data from this experiment were compared to the results of the former GAS M1T1 study, and to predictions of two recently published bioinformatics tools [19,21]. Additionally, we tested the regulation of sRNA expression in correlation to growth media and growth phase. We found very little overlap between the different screening methods, which underlines the importance of using several complementary methods, as well as several environmental conditions, to attain a comprehensive analysis of bacterial sRNAomes.

\section{Results}

Identification of sRNAs in the intergenic regions of GAS M49 Custom intergenic tiling arrays representing the genome of S. pyogenes NZ131 (NCBI accession number: NC_011375) were designed to detect the expression of potential sRNAs. A total of 17,823 50-mer probes with an overlap of $15 \mathrm{bp}$ were synthesized to cover the intergenic regions, with 9,082 probes representing the positive strand and 8,741 probes covering the negative strand. Additionally, 174 probes were designed as control probes covering tRNA genes or genes coding for known sRNAs. For example, we probed for fasX [17], SR914400, and SR1754950 [20], all of which were detected in our experiments. Genedata Selector software was used to integrate genomes, tiling array probe sequences, sRNA predictions, and experimental data. Expression data were analysed in their genomic- and sequence-based contexts (Genedata AG).

Total RNA for the tiling array experiments was isolated from GAS M49 grown in chemically defined medium (CDM). Samples were taken from four biological replicates during both mid-log growth phase $\left(\mathrm{OD}_{600}=0.4-0.6\right)$ and stationary growth phase $\left(\mathrm{OD}_{600}=\right.$ 1.2). A signal intensity of $>300$ was set as a threshold. A positive signal required that a minimum of one probe specific for one strand showed an intensity above the threshold in at least three replicates. Intergenic regions featuring high intensities on both strands were manually removed following the analysis.

We identified a total of 55 putative sRNAs in GAS M49 that were expressed during growth in CDM, 42 of which were novel. Computational functional prediction revealed that a subset of the newly identified RNAs included molecules with similarities to one of the common non-coding RNA families included in the Rfam database [22]. The database covers functional categories of non-coding RNAs determined from multiple sequence alignments. Using the Rfam database, we predicted functions for 14 putative sRNAs. One of these RNAs was predicted to be the structural RNA of the bacterial signal recognition particle (SRP), and another was predicted to be the bacterial RNase P RNA. Further functional categories included three T-box leader elements, three CRISPR family members, one tmRNA, and one endoribonuclease, RNaseP_bact_b (Table 1). Table 1 contains a summary of the 
Table 1 S. pyogenes M49 sRNAs, genomic location, Rfam predictions, and conservation across streptococcal genomes

\begin{tabular}{|c|c|c|c|c|c|c|c|c|c|c|}
\hline No & ID & $\begin{array}{l}\text { Left } \\
\text { nucleotide }\end{array}$ & $\begin{array}{l}\text { Right } \\
\text { nucleotide }\end{array}$ & Size (nt) & Strand & $\begin{array}{l}\text { Upstream } \\
\text { adjacent } \\
\text { gene }\end{array}$ & $\begin{array}{l}\text { Downstream } \\
\text { adjacent } \\
\text { gene }\end{array}$ & $\begin{array}{l}\text { Rfam and } \\
\text { MOSES } \\
\text { prediction }\end{array}$ & $\begin{array}{l}\text { Previously } \\
\text { published }\end{array}$ & Conservation \\
\hline 1 & sRNASpy490131 & 141429 & 141478 & 49 & - & ntpK & ntpE & & & a \\
\hline 2 & sRNASpy490186c & 194707 & 194791 & 84 & + & Spy49_0186c & speG & SRP_bact & {$[37]$} & c \\
\hline 3 & sRNASpy490206 & $216807^{d}$ & 217021 & 215 & $+^{e}$ & fas $A$ & rnpA & MOSES2 & $\operatorname{fas} X[17]$ & $b$ \\
\hline 4 & sRNASpy490229 & 238850 & 238899 & 49 & + & $\operatorname{prgA}$ & rpsL & & & $b$ \\
\hline 5 & sRNASpy490238 & 248530 & 248579 & 49 & + & Spy49_0238 & bacA & & & a \\
\hline 6 & sRNASpy490241 & 251595 & 251644 & 49 & + & $\operatorname{rgpG}$ & Spy49_0242 & & & a \\
\hline 7 & sRNASpy490305 & 318826 & 318910 & 84 & + & Spy49_0305 & Spy49_0306 & 23S-methyl & & $b$ \\
\hline 8 & sRNASpy490306 & 319622 & 319776 & 154 & + & Spy49_0306 & Spy49_0307 & FMN & RNA319780 [20] & $b$ \\
\hline 9 & sRNASpy490348 & 362857 & 362906 & 49 & + & Spy49_0348 & Spy49_0349c & MOSES6 & & a \\
\hline 10 & sRNASpy490366c & 373806 & 373855 & 49 & + & Spy49_0366c & Spy49_0367c & & & - \\
\hline 11 & sRNASpy490370 & 376843 & 376892 & 49 & + & Spy49_0370 & Spy49_0371 & & & a \\
\hline 12 & sRNASpy490380c & $385431^{d}$ & 385526 & 95 & $++^{e}$ & Spy49_0380c & $\mathrm{mtsA}$ & & & $b$ \\
\hline 13 & sRNASpy490388 & 393522 & 393571 & 49 & + & rplA & pyrH & & & a \\
\hline 14 & sRNASpy490434 & 431752 & 431836 & 84 & + & Spy49_0434 & thrs & T-box & & $b$ \\
\hline 15 & sRNASpy490483c & $476503^{d}$ & 476600 & 97 & $+{ }^{e}$ & Spy49_0483c & bglG & & & b \\
\hline 16 & sRNASpy490493 & 486133 & 486182 & 49 & + & Spy49_0493 & ptsK & & & a \\
\hline 17 & sRNASpy490504 & 495351 & 495400 & 49 & - & Spy49_0504 & Spy49_0505c & & & a \\
\hline 18 & sRNASpy490592 & 594852 & 594936 & 84 & + & Spy49_0592 & phes & & & $\mathrm{b}$ \\
\hline 19 & sRNASpy490727 & 733841 & 733890 & 49 & - & Spy49_0727 & Spy49_0728 & & & a \\
\hline 20 & sRNASpy490822 & 820820 & $820996^{d}$ & 176 & - e & Spy49_0822 & Spy49_0823 & CRISPR & RNA772970 [20] & c \\
\hline \multirow[t]{2}{*}{21} & sRNASpy490827 & 827456 & 827785 & 329 & + & Spy49_0827 & Spy49_0828 & CRISPR & & \\
\hline & & & & & & & & MOSES14 & & a \\
\hline 22 & sRNASpy490845c & 846977 & 847061 & 84 & + & trmE & rplJ & L10_leader & & c \\
\hline 23 & sRNASpy490948c & 941583 & $941657^{d}$ & 74 & - & Spy49_0948c & guaA & & & a \\
\hline 24 & sRNASpy490957c & 950434 & 950518 & 84 & - e & Spy49_0957c & Spy49_0958c & & SR914400 [20] & $b$ \\
\hline 25 & sRNASpy491007c & 1000709 & 1000828 & 119 & + & pcrA & Spy49_1008 & Glycine & & $b$ \\
\hline 26 & sRNASpy491023c & 1019382 & 1019746 & 364 & + & Spy49_1023c & Spy49_1024 & tmRNA & RNA983400 [20] & $b$ \\
\hline 27 & sRNASpy491061c & 184794 & 184878 & 84 & + & Spy49_1061c & Spy49_0176 & & & c \\
\hline 28 & sRNASpy491095c & 1092894 & 1092943 & 49 & - & ptsH & $\mathrm{nrdH}$ & & & c \\
\hline 29 & sRNASpy491122c & 1117169 & 1117218 & 49 & - & sodM & holA & & & $b$ \\
\hline 30 & sRNASpy491163c & 1156488 & 1156537 & 49 & - & Spy49_1163c & $\mathrm{clpE}$ & & & a \\
\hline 31 & sRNASpy491167c & 1163270 & 1163319 & 49 & - & iles & divIVA & T-box & & - \\
\hline 32 & sRNASpy491206c & 1201615 & 1201734 & 119 & - & $\operatorname{ccdA}$ & Spy49_1207c & MOSES16 & RNA1239700 [20] & a \\
\hline 33 & sRNASpy491217c & 1214010 & 1214059 & 49 & - & Spy49_1217c & Spy49_1218c & & SR1251900 [20] & $\mathrm{b}$ \\
\hline 34 & sRNASpy491243 & 1246370 & 1246419 & 49 & + & Spy49_1243 & Spy49_1244 & & & a \\
\hline 35 & sRNASpy $491275 c$ & 1282084 & 1282413 & 329 & - & Spy49_1275c & Spy49_1276c & RNaseP_bact b & RNA1320100 [20] & c \\
\hline 36 & sRNASpy491311c & 1321638 & $1321853^{d}$ & 84 & - e & glyQ & Spy49_1312c & & & $b$ \\
\hline 37 & sRNASpy491336c & 1341554 & 1341603 & 49 & + & rbfa & $\operatorname{infB}$ & & & $b$ \\
\hline 38 & sRNASpy491340c & 1346300 & 1346349 & 49 & - & nus A & Spy49_1341c & & & $b$ \\
\hline 39 & sRNASpy491354 & 1357803 & 1357852 & 49 & - & manL & $\operatorname{manM}$ & & & $b$ \\
\hline 40 & sRNASpy491357 & 1360204 & 1360323 & 119 & + & Spy49_1357 & sers & T-box & RNA1439100 [20] & $\mathrm{b}$ \\
\hline 41 & sRNASpy491409c & 1413359 & 1413408 & 49 & - & acps & $\sec \mathrm{A}$ & & & a \\
\hline 42 & sRNASpy491489c & 1489011 & 1489060 & 49 & + & Spy49_1489c & Spy49_1491c & & & $b$ \\
\hline
\end{tabular}


Table 1 S. pyogenes M49 sRNAs, genomic location, Rfam predictions, and conservation across streptococcal genomes (Continued)

\begin{tabular}{|c|c|c|c|c|c|c|c|c|c|c|}
\hline 43 & sRNASpy491555c & 1528842 & 1528891 & 49 & - & Spy49_1555c & Spy49_1556c & & & a \\
\hline 44 & sRNASpy491560c & 1533843 & 1533892 & 49 & - & Spy49_1560c & pyrG & & & a \\
\hline 45 & sRNASpy491561c & 1535755 & 1535804 & 49 & - & pyrG & rpoE & & & c \\
\hline 46 & sRNASpy491562c & 1536613 & 1536662 & 49 & - & rpoE & tig & & & a \\
\hline 47 & sRNASpy491591 & 1571896 & 1571945 & 49 & - & lacR2 & Spy49_1592 & MOSES18 & SR1604140 [20] & a \\
\hline 48 & sRNASpy491596c & 1574723 & 1574807 & 84 & - & rplM 505 & Spy49_1597c & L13_leader & & C \\
\hline 49 & sRNASpy491671c & 1654859 & 1654908 & 49 & - & emm49 & Spy49_1672c & & & a \\
\hline 50 & sRNASpy491707c & 1693440 & 1693489 & 49 & - & Spy49_1707c & Spy49_1708 & & & a \\
\hline 51 & sRNASpy491713 & 1698142 & 1698191 & 49 & + & Spy49_1713 & Spy49_1714c & & SR1719800 [20] & a \\
\hline 52 & sRNASpy491718c & 1704471 & 1704525 & 54 & - & $\mathrm{cts} R$ & $\operatorname{csp}$ & & & a \\
\hline 53 & sRNASpy491732c & 1724321 & 1724370 & 49 & + & Spy49_1732c & rpsB & & SR1745900 [20] & $b$ \\
\hline 54 & sRNASpy491738 & 1733300 & $1733433^{d}$ & 133 & - & Spy49_1738 & Spy49_1739c & & SR1754950 [20] & $b$ \\
\hline 55 & sRNASpy491769c & 1763496 & 1763545 & 49 & + & hisS & rpmF & & & $b$ \\
\hline
\end{tabular}

Coordinates and gene designations of adjacent genes are relative to the S. pyogenes NZ131 genome (NCBI accession number: NC_011375).

a Streptococcus pyogenes.

b Beta-haemolytic streptococci.

' Non-beta-haemolytic streptococci.

${ }^{d}$ Transcriptional start site determined by $5^{\prime}$ RACE.

${ }^{\text {e }}$ Strand confirmed by gene specific RT-PCR.

information pertaining to all 55 candidate sRNAs, including the flanking genes, Rfam prediction, and conservation across other genomes. Five putative sRNA sequences overlapped with adjacent ORFs on the same strand. Functional studies will be necessary to clarify whether the corresponding sRNAs are transcribed independently. The overall GC content of all 55 sRNA candidate sequences was $38.3 \%$. This correlates with the GC content of the whole NZ131 genome (38.0\%). No specific strand prevalence and no clustering in specific genomic regions were observed for the regulatory sRNA genes. The replication-related gene orientation bias of the protein coding genes [23,24] was mirrored by the sRNA gene candidates. A circular depiction was created with the Artemis DNAPlotter tool [25] (Figure 1) to visualize the sRNA genes in the context of the NZ131 genome.

In a previous bioinformatic approach (MOSES), 20 probable candidates were predicted [19]. In our array analysis, expression of five of the predicted sRNAs was confirmed under the conditions studied (Table 1). Furthermore, we detected 12 streptococcal RNAs that were previously identified by Perez et al. [20] (Table 1). The phylogenetic conservation of the putative sRNAs was tested by BLAST analysis, and the taxonomic classification was presented following the nomenclature of Facklam [26]. The tiling array technique with overlapping probes did not allow us to detect an accurate start site for the respective sRNA genes. Thus, we described the nucleotides represented by the active probes as the preliminary start and end (Table 1).

For a subset of six candidate sRNA genes, we determined the transcriptional start site (TSS) of the sRNA molecules using the $5^{\prime}$ rapid amplification of cDNA ends- ( $5^{\prime}$ RACE) technology (Invitrogen). The TSS of the analysed sRNAs is shown in Table 1. $5^{\prime}$ RACE was conducted for two known sRNA genes, fas $X$ and sRNASpy490822 (CRISPR1), for one candidate predicted by MOSES (MOSES4), and for three novel sRNA candidates, sRNASpy490483c, sRNASpy491311c, and sRNASpy491738. The results of the $5^{\prime}$ RACE analysis are shown in Figure 2. Promoter and terminator predictions for the respective sRNA candidate genes are also included.

\section{Comparison of the sRNA expression data using different sRNA screens}

The tiling array data were compared with the prediction results of two recently published bioinformatics tools, sRNAScanner [21] and MOSES [19]. As shown in Figure 3A, the overlap between the GAS M49 array data and the sRNA predictions performed with the sequence of the NZ131 genome was minimal. From 20 MOSES candidates, five were detected in the tiling array analysis, while from 137 sRNAScanner predictions, 11 showed a signal in the array. Eight putative sRNAs were selected by both programs. There was only one sRNA that was predicted by both algorithms and was also detected in the tiling array (Figure 3A). The general accuracy of the three independent screens was supported by the fact that this mutual candidate was the known streptococcal sRNA FASX [17].

We also compared our tiling array data with a previously published sRNA microarray study in GAS M1T1 [20]. The previous study identified 40 putative sRNAs. 


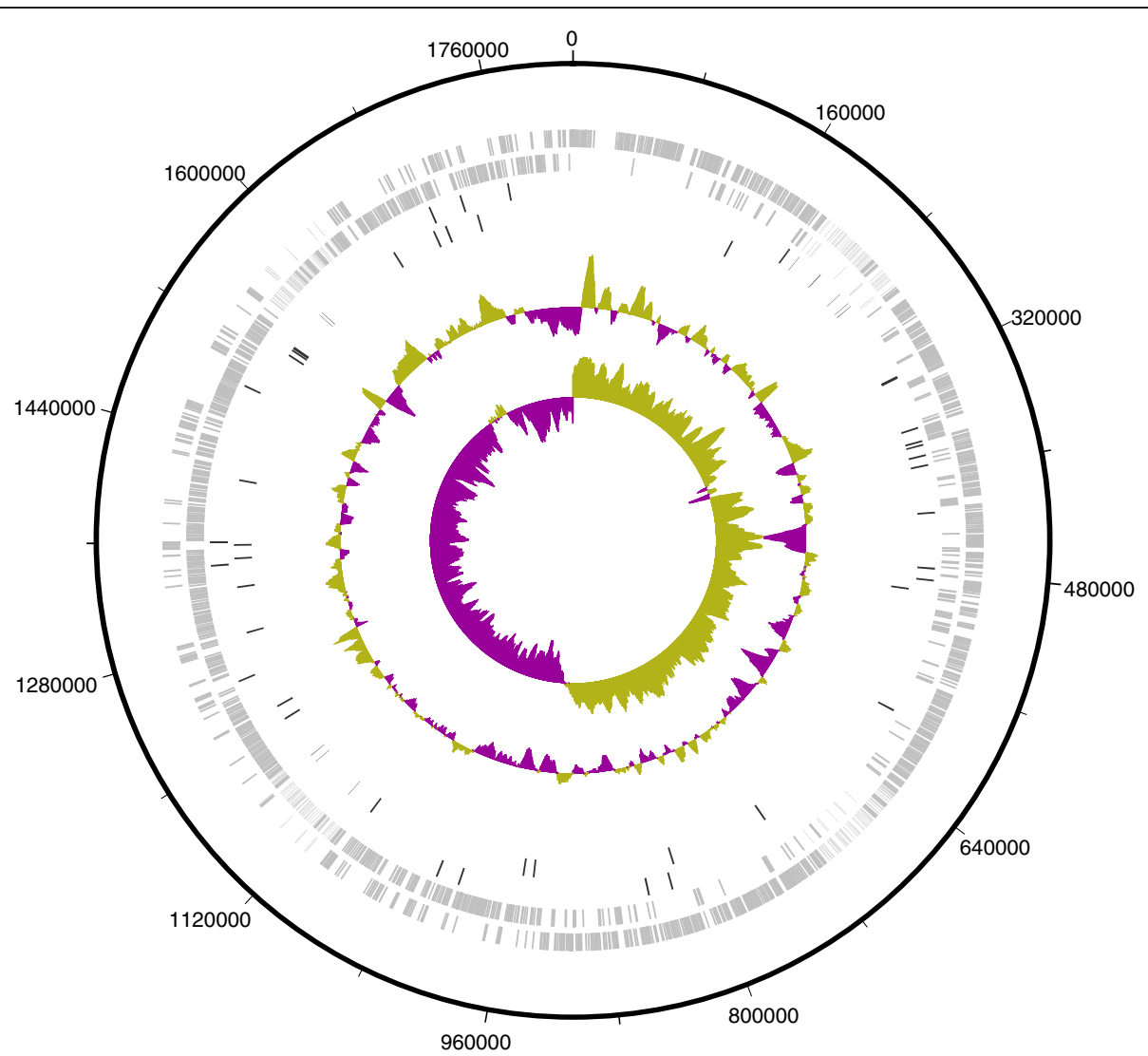

Figure 1 Circular representation of the GAS M49 genome. Tracks from outermost moving inward: Track 1, NZ131 genome; Tracks 2 and 3 , presence of coding genes on the forward and reverse strand, respectively; Tracks 4 and 5, sRNA genes forward and reverse, respectively. Tracks 6 and 7 show the GC plot and the GC skew of the genome.

Even though the sequences of the candidates were conserved across streptococcal genomes, serotype-specific variation of sRNA transcript abundance was observed in northern blot experiments. Screening of GAS M1T1 was conducted with cells grown in complex medium [20], whereas the expression experiments in this study were performed with GAS M49 grown in CDM. Consequently, we found only a limited overlap between the two microarray screens (Figure 3B). Twelve sRNA candidates were detected in both strains.

\section{Analysis of common motifs in the GAS M49 sRNA population}

To identify putative functional regions within the sRNA candidates, all sequences were screened for common features by motif-based sequence analysis using MEME SUITE [27]. The occurrence of shared sequence motifs in different sRNA species could be an indication of common structural properties with functional significance in this region. Seven motifs were identified with consensus sequences with $p$-values $<1.0 \times 10^{-7}$, spanning $9-27$ base pairs (Figure 4). Putative motif sequences were compared to members of the Rfam database families, and were subjected to TOMTOM [28] motif analysis using the RegTransBase prokaryotic database. Candidates sRNASpy490592 and sRNASpy491336c shared a 14 bp consensus sequence with no apparent known function (motif 4, Figure 4). For all other identified motifs, a known function could be assigned to the respective candidates. The corresponding RNAs were either predicted to be RNAs with non-regulatory functions, e.g. RNAseP (motif 5, Figure 4), or more typically, to represent cis-regulatory RNA elements, e.g. FMN riboswitch or MET box (motif 6 and motif 7, Figure 4).

\section{Regulated expression of sRNA genes in GAS M49}

Regulation of sRNA gene expression in GAS M49 under different growth conditions was studied by intergenic tiling array analyses. Total RNA was isolated from bacteria grown in CDM, BHI, or THY. Samples were collected in the exponential and stationary phases. Transcript level changes were expressed as the $\log _{2}$ signal ratio between conditions, and are listed in Table 2. sRNA gene expression was considered significantly different when the $\log _{2}$ ratio of the signals was $\leq-1.58$ or $\geq 1.58$. Twenty-four sRNA genes were regulated in a growth phase- and/or medium- 


\begin{abstract}
FASX
RNA: 216807-217021, $215 \mathrm{nt}$

atgatgctcttattgaaacatttttcgctataataattaatgtaaataaagatttacgaagtcatgagtttatcgagcaatca atccccaattcaatcaaatattgttttctctctctccataaacaaatagtatttttgatatgatggctcggcagacaaaatg acgatgtcagttgtctttgtttggatatcacattagctgattgtaaatcagtattaaagcccggcttgccgggtttttgat aggtaa

MOSES4

RNA: 308505-308758, $254 \mathrm{nt}$

gatttcttgctttattttaaatgaataagtctgatacgtttccttccttaacactatcgactccataatgacccatcagtcaaac ggtatatctcaaacgactacggtttactccaaattgattggctagttgaggccaagaaacacctgattttttaatgctaaattt ctagcttatcttcatagct taattcatacaaaaaaccccaatcgttagattttttgatatgtccctttcactagatgagtc cagtaaagggggcatatcgtttttgtctaactt
\end{abstract}

\title{
SRNASpy490483c
}

RNA: 476503, no terminator prediction

ttgacagaatcttaaagagatgataacattaaagcgttctcagatgtgattacctcaggtaagcaggctagagacataacaat cagaatattaaaggatgttatcacagttttgtgagcagggacctattcgaaaagtgcttttagcgagcttttggataggtca ttttttattgtgatactaaaggactaggacttttatgttgattaaacgcgttttaaaccataatg

\section{sRNASpy490822 (CRISPR1)} RNA: 820820-820996, $176 \mathrm{nt}$

aaatccttttaaaagtagtttattttgttatcattctatagtattaagtattgttttatggctgataaattttttgaattt ctccttgattatttgttataaaagttataaaataatcttgttggaaccattcaaaacagcatagcaagttaaaataaggctag tccgttatcaacttgaaaagtggcaccgagtcggtgcttttttgatacttc

\section{SRNASpy491311c}

RNA: 1321853-1321638, $215 \mathrm{nt}$

tggacaaactaatggctttatgctaaactagactctaggaaaaaggatgcaagtatcttatctaaagcgagttcggggtagtt agagccgaatggtaggactgcagattggcgcttccgtttgggcagtgtgattaagtatatttgtcaatattgcccaaaagat actatataaatgaagtaataaattagggtgggaaccgcgttttgacgcccctaggtcttatgatttaggagcatcaaaatgcg g

\section{sRNASpy491738}

RNA: 1733433-17333300, 133 nt

ttgcgctcatctcacgaatatgataatataatatcaagtctgtttgagaaaacagaagctcaggtcaagtaagtgcacactta caataacacagttgataatggggaaactcagagtcaagaaaagaaaagcaagccttgcattttgcaaggcttttttctgtct

caatar

Figure 2 Determination of the transcriptional start site of putative sRNAs. Transcriptional start sites were detected by $5^{\prime}$ RACE-PCR and are depicted in black/bold. The sequenced sRNA sequence is depicted in black, predicted RNA sequences are depicted in grey, predicted promoter regions are depicted in blue, predicted rho-independent transcription terminators are depicted in green. The start codon of down-stream genes is depicted in orange.

dependent fashion. During growth in CDM, five genes were up-regulated in the stationary growth phase compared to the exponential growth phase, whereas three genes were down-regulated. One of the down-regulated sRNA genes was fas $X$. This is in accord with previous results, where a reduction in fas $X$ transcript abundance in the stationary growth phase was detected by northern blot analysis [17]. This observation was also confirmed by qRT-PCR analyses (Figure 5B). Comparison of sRNA gene expression during growth in THY with expression during growth in CDM revealed differential expression of 17 sRNA genes. Of these, 13 genes were down-regulated and four up-regulated in THY. Growth in BHI led to the detection of 12 mediadependent controlled sRNA genes. Nine genes were downregulated and three were up-regulated during growth in BHI compared to growth in CDM. From the 20 sRNA genes that showed media-dependent regulation, seven were regulated in both THY and BHI, and showed the same direction of regulation compared to CDM. Twelve sRNA genes were exclusively regulated in one of the two media, and only one gene was down-regulated in THY but upregulated in $\mathrm{BHI}$ compared to CDM. These results are in accord with the fact that both media THY and BHI are complex media, as opposed to the synthetic medium CDM, which forces the bacteria to synthesize a number of components essential for growth. Thus, in CDM, changes in bacterial metabolism are necessary for successful growth and require adaption of the bacterial transcriptome, including the sRNAome.

\section{Validation of sRNA expression by qRT-PCR and northern blot analyses}

Expression of sRNA candidates by GAS M49 was tested by gene-specific reverse transcription followed by realtime PCR analysis. Experimental expression validation was performed for the RNAs FASX and sRNASpy490822 (CRISPR1), for the sRNA scan7, which was predicted using sRNAscanner [21], and for three more candidates 


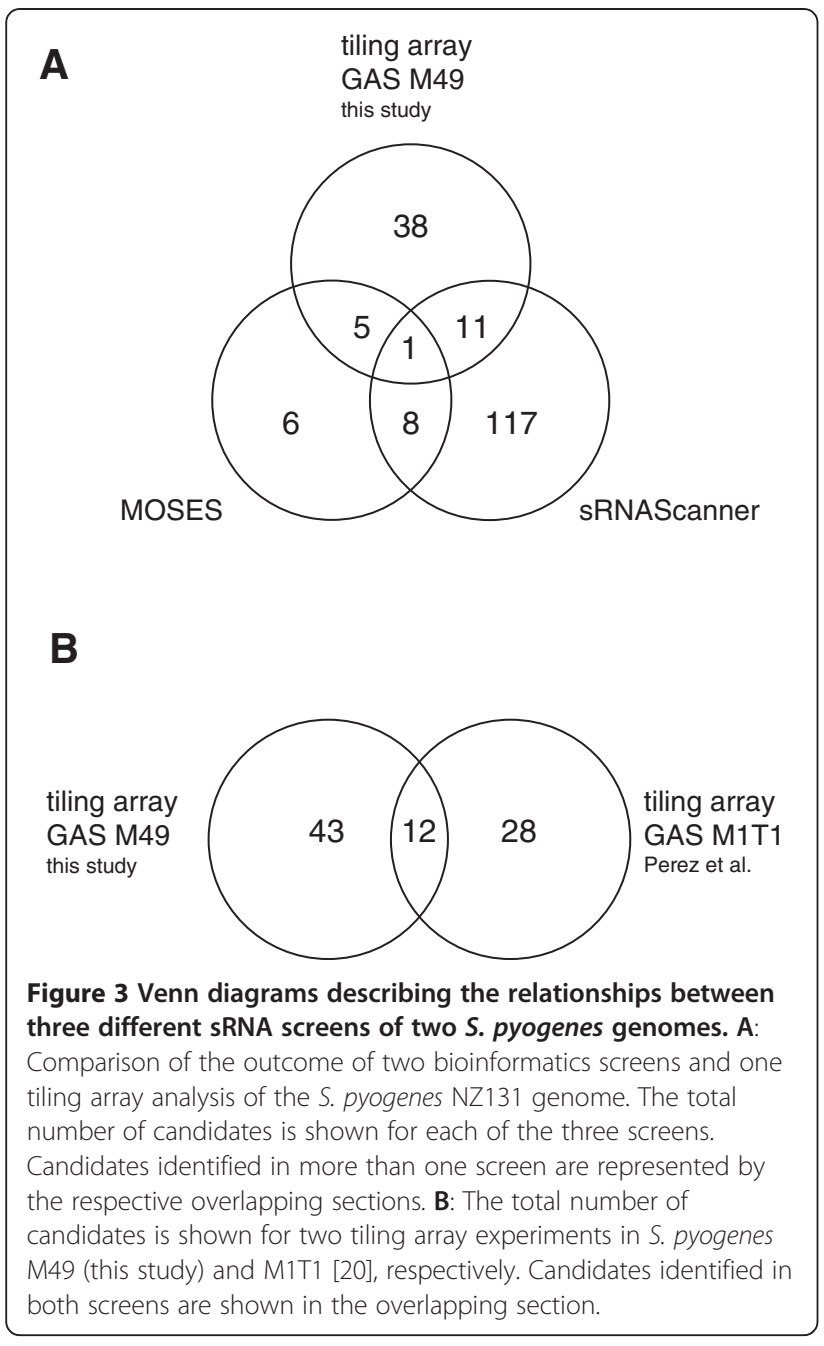

identified by tiling arrays in this study (sRNASpy490380c, sRNASpy490957c, and sRNASpy491311c). The expression of the candidates in GAS M49 was verified. Moreover, we confirmed the orientation of the sRNA genes by employing single gene-specific primers for the reverse transcription reaction. Three reactions were performed in parallel: one including the forward primer, one including the reverse primer, and one without any primers. Signals were only detected in samples containing the primer complementary to the coding strand of the respective gene (data not shown). We compared sRNA expression of GAS M49 cultured in CDM medium and THY broth throughout growth (Figure 5). 5S RNA was used as an internal control for normalization, and was expressed in comparable amounts under all conditions tested in this experiment. Expression of fas $X$ was equivalent during growth in CDM and THY (Figure 5A). FASX was down-regulated in the stationary phase, an observation that confirmed the array data and previously published results from northern blot analyses [17]. We did not detect strong regulation of CRISPR1, sRNASpy490957c, or sRNASpy491311c during growth in
CDM (Figure 5B). In contrast, scan7 was highly upregulated in the stationary phase (Figure $5 \mathrm{~B})$. The expression of sRNASpy490380c was much higher in THY compared to CDM (almost 100-fold, data not shown). During growth in CDM, no changes in the low level expression of sRNASpy490380c were observed (data not shown).

To further verify candidate gene expression, northern blot analysis of the same putative sRNA genes was performed (Figure 6). This method allows the determination of approximate transcript sizes. Probes specific for 5S RNA and FASX were included as controls. The apparent molecular weight of candidates CRISPR1, sRNASpy490380c, sRNASpy490483c, sRNASpy491311c, and scan7 corresponded to the length predicted by $5^{\prime}$ RACE determination. The CRISPR transcript, tracrRNA, showed a band at the expected size of 176 nucleotides, as well as several smaller bands that were likely the result of RNA processing, as observed previously in GAS M1T1 [29] (Additional file 1A). For the putative sRNASpy490957c, transcript analysis by $5^{\prime}$ RACE predicted a 161 nt full-length product, including the terminator region. However, the most prominent band detected by northern blot analysis migrated at approximately $80 \mathrm{nt}$. Low intensity bands were detected at approximately $90 \mathrm{nt}$ and $160 \mathrm{nt}$ (Additional file 1B), which might indicate post-transcriptional sRNA processing.

\section{Discussion}

Bacterial gene regulation by sRNAs has gained a lot of attention in recent years, because it plays an important role in many cellular processes, including response to environmental changes, growth, and pathogenesis. There is an intriguingly large diversity of regulatory mechanisms, including cis- and trans-acting sRNAs, untranslated regions, and riboswitches. Some sRNA molecules act as repressors of translation and destabilize mRNA transcripts, but others act by activating and stabilizing target mRNAs [30-32]. One of the best characterized sRNAs in GAS is FASX, which is involved in virulence-related gene regulation $[17,33]$. Knock-out mutants of fas $X$ show a reduced expression of secreted virulence factors such as streptokinase and streptolysin $\mathrm{S}$. The mechanism for streptokinase gene $(s k a)$ expression control is the stabilization of the $s k a$ transcript [33]. Lack of FASX-ska-mRNA-interaction in the fas $X$ deletion mutant decreased transcript levels, and consequently decreased streptokinase protein abundance.

A second example of a regulatory RNA in GAS is the untranslated mRNA of the streptococcal pleiotropic effect locus (pel), which contains sagA, the structural gene for streptolysin $\mathrm{S}$. This region was described as a positive regulator of important streptococcal virulence factors, including M-protein, Sic, and SpeB [34]. Strain specificity of PEL function is indicated by the fact that emm transcription was not affected in a sagA-deficient mutant with a M6 background [35]. Similar results have 


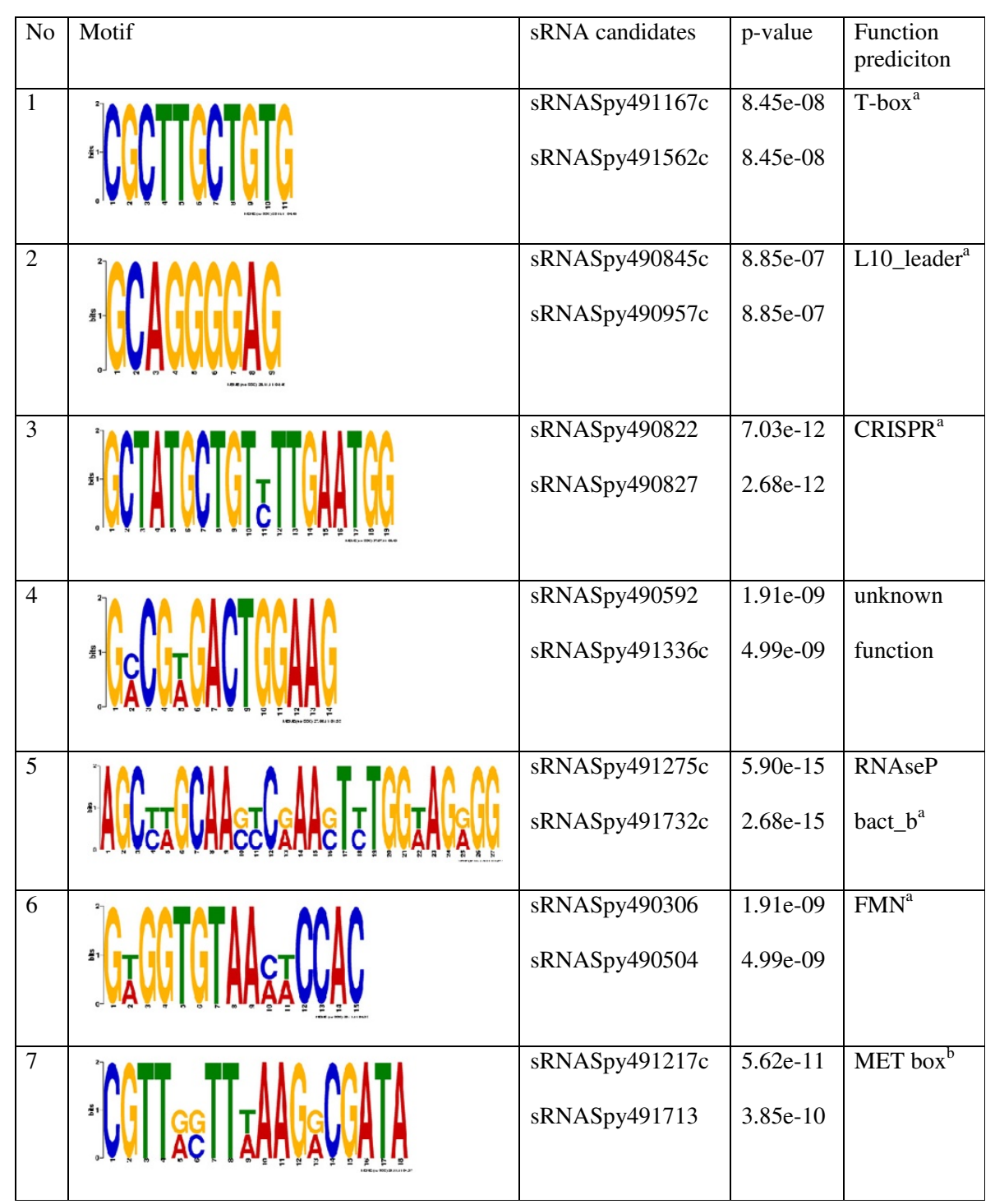

Figure 4 Shared sequence motifs in sRNA candidates. ${ }^{a}$ Rfam, ${ }^{\text {'TOMTOM. }}$

been obtained in GAS M1 and M18 Tn916 sagA mutant strains [36]. Additionally, pel deletion mutant analysis of four M1T1 GAS isolates did not identify any regulatory function for the pel sRNA in this serotype [20].

Another, more recently described untranslated RNA with influence on streptococcal virulence is the $4.5 \mathrm{~S}$ RNA, a component of the bacterial signal recognition particle (SRP) [37]. While the 4.5S RNA gene is not essential, mutation impairs bacterial growth, lowers virulence factor secretion, and reduces virulence in a mouse infection model.

Recently, several whole genome sRNA screens in Gram-positive bacteria, employing either tiling array or next generation sequencing approaches, revealed an unexpected number of potential sRNAs in several pathogenic species [38-42]. In this context, it is likely that GAS expresses more sRNAs responsible for virulence gene expression control. One whole-genome intergenic tiling array screen of GAS M1T1 identified approximately 40 sRNAs that were expressed during the exponential growth phase in cells cultivated in THY complex medium [20]. The GAS M49 sRNAome in the present study was determined using cells grown in CDM. From 55 putative sRNAs in GAS M49, only 12 were detected previously in the GAS M1T1 screen (Figure 3B). This result is in accord with the concept that sRNA expression is serotype-dependent and regulated by environmental stimuli. Consequently, we detected media- and growth-phase-dependent sRNA gene regulation in the tiling array expression analysis, or by qRTPCR of selected candidate genes. It would be interesting to monitor sRNA gene expression regulation under infectionrelevant conditions. 
Table 2 Regulation of S. pyogenes M49 sRNA gene expression dependent on culture medium and growth phase

\begin{tabular}{|c|c|c|c|c|c|c|}
\hline No & ID & $\begin{array}{l}\text { THY vs CDM } \\
\text { exponential } \\
\text { growth phase }\end{array}$ & $\begin{array}{l}\text { THY vs CDM } \\
\text { stationary } \\
\text { growth phase }\end{array}$ & $\begin{array}{l}\text { BHI vs CDM } \\
\text { exponential } \\
\text { growth phase }\end{array}$ & $\begin{array}{l}\text { BHI vs CDM } \\
\text { stationary } \\
\text { growth phase }\end{array}$ & $\begin{array}{l}\text { Stationary vs } \\
\text { exponential } \\
\text { in CDM }\end{array}$ \\
\hline 1 & sRNASpy490186c & & $-2,72$ & & & \\
\hline 2 & sRNASpy490206 & $-1,78$ & & & & $-2,00$ \\
\hline 3 & sRNASpy490238 & 3,33 & & & & \\
\hline 4 & sRNASpy490305 & $-2,50$ & $-3,03$ & $-2,10$ & $-3,59$ & \\
\hline 5 & sRNASpy490306 & & & & 1,72 & \\
\hline 6 & sRNASpy490348 & 3,42 & & & & \\
\hline 7 & sRNASpy490370 & & & & $-2,51$ & \\
\hline 8 & sRNASpy490380c & & $-3,02$ & $-2,28$ & $-3,82$ & \\
\hline 9 & sRNASpy490493 & 2,18 & & & & \\
\hline 10 & sRNASpy490592 & & & & & 1,81 \\
\hline 11 & sRNASpy490827 & $-1,89$ & & & & \\
\hline 12 & sRNASpy490845c & & & & $-1,98$ & 2,03 \\
\hline 13 & sRNASpy490957c & & $-2,67$ & & & \\
\hline 14 & sRNASpy491007c & & $-1,73$ & & $-1,81$ & 1,94 \\
\hline 15 & sRNASpy491023c & $-1,84$ & & 1,92 & 3,54 & \\
\hline 16 & sRNASpy491206c & 1,58 & & & 2,65 & $-2,41$ \\
\hline 17 & sRNASpy491311c & & & & & $-2,20$ \\
\hline 18 & sRNASpy491561c & $-1,99$ & & & & \\
\hline 19 & sRNASpy491596c & & $-1,60$ & $-1,88$ & & \\
\hline 20 & sRNASpy491707c & & & & & 1,68 \\
\hline 21 & sRNASpy491713 & & & & & 1,76 \\
\hline 22 & sRNASpy491718c & & & $-1,71$ & & \\
\hline 23 & sRNASpy491732c & & $-2,38$ & & $-3,60$ & \\
\hline 24 & sRNASpy491738 & & $-3,40$ & $-2,07$ & $-4,30$ & \\
\hline
\end{tabular}

Clustered, regularly interspaced, short palindromic repeat (CRISPR) loci represent an adaptive RNA-based immune system that protects bacteria and archaea from horizontal transfer of phage and plasmid DNA [43]. Among the putative sRNA genes detected in GAS M49 by the tiling array approach, two sequences were categorised by the Rfam prediction program as CRISPR-related RNAs (Table 1). sRNASpy490822 and sRNASpy490827 are encoded by the system II (Nmeni/CASS4 subtype) [44] CRISPR/Cas locus, which was characterized recently by differential RNA sequencing in GAS SF370 (M1 serotype) [29]. Our data suggest that this locus is also active in GAS M49. Expression of sRNASpy490822 was confirmed by RT-PCR on the opposite strand of the CRISPR-associated genes under all conditions tested in this study. This transcript corresponds to the trans-activating CRISPR RNA (tracrRNA), which is responsible for the maturation of CRISPR RNA in concert with RNase III and the CRISPRassociated Csn1 protein [29]. A third CRISPR-related RNA detected in our expression screen, sRNASpy491206c, is encoded in the system I-C (Dvulg/CASS subtype) [44]
CRISPR/Cas locus, which is also conserved in streptococcal genomes. In contrast to our array data, this locus appeared to be silent in GAS SF370, where no expression was detected in the differential RNA sequencing approach [29]. Even though the CRISPR loci are conserved throughout GAS genomes, the activity of different CRISPR subtypes appears to be serotype-specific.

In the early years of sRNA research, many bioinformatic prediction tools were developed. One of the most prominent programs was the SIPHT tool, which has been used for many bacterial species $[45,46]$. However, comparison of the prediction results with the actual in vivo expression of sRNAs often revealed very little overlap between the different screening methods $[20,41,47]$. The reasons for this discrepancy may be the limitations of the prediction programs as well as the fact that not all sRNAs are expressed under all conditions. The development of sRNA prediction software with improved properties is on-going. We compared our tiling array data with the prediction results of two recently published bioinformatics tools, sRNAScanner [21] and 

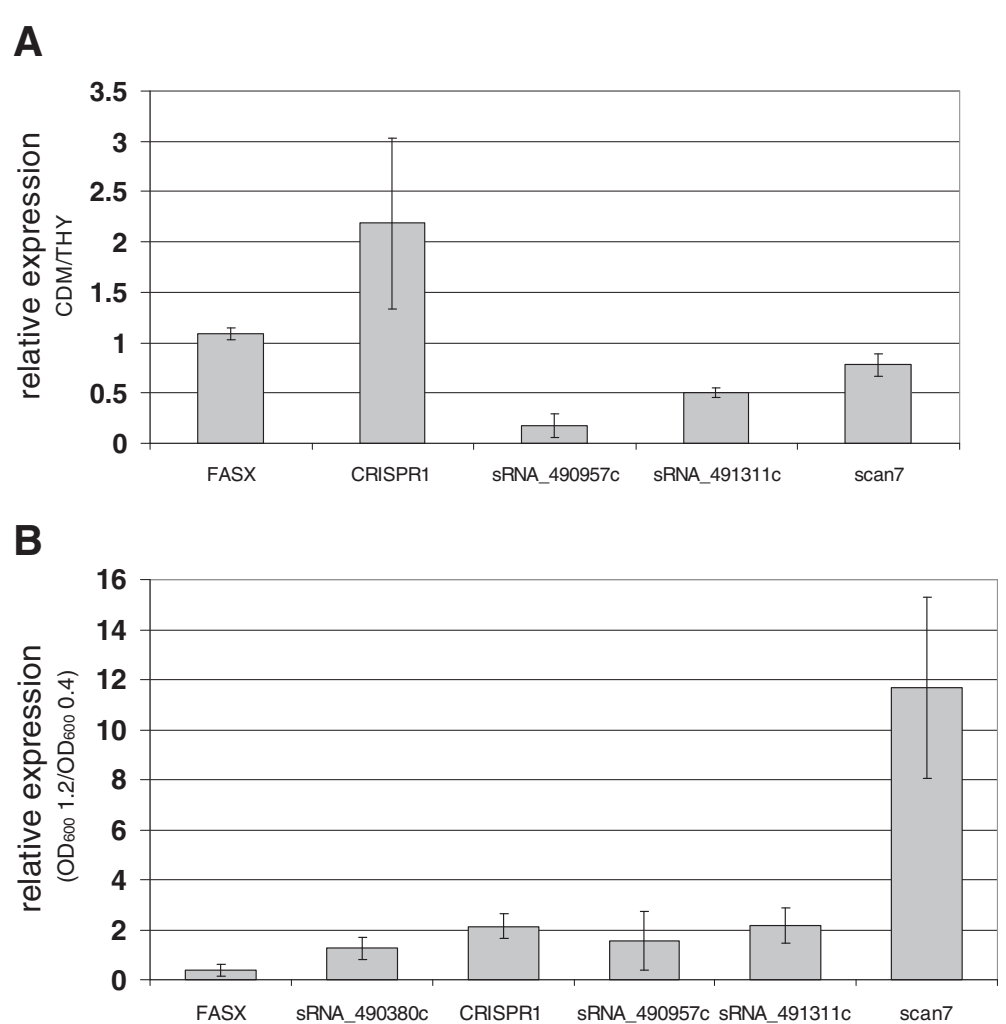

Figure 5 Expression of sRNA candidates verified by qRT-PCR analysis. A: sRNA expression during growth in CDM compared to growth in THY. Samples were taken during the exponential growth phase $\left(\mathrm{OD}_{600}=0.4\right)$. Relative sRNA gene expression in $C D M$ is depicted in this graph (expression in THY is normalised to one). B: sRNA gene expression throughout growth in CDM. Samples were taken in the exponential growth phase $\left(\mathrm{OD}_{600}=0.4\right)$ and in the stationary phase $\left(\mathrm{OD}_{600}=1.2\right)$. Relative sRNA gene expression at $\mathrm{OD}_{600}=1.2$ is depicted in this graph $\left(\mathrm{OD}_{600}=0.4\right.$ is normalised to one $)$.

MOSES [19]. As depicted in Figure 3A, the overlap between the tiling array expression data and the sRNA predictions was low. From the 20 most probable candidates of the MOSES analysis, 25\% were expressed in GAS M49, whereas $8 \%$ of the predicted sRNAScanner predictions were found in the array analysis. Even the overlap between the two bioinformatics data sets was low. The only sRNA that was detected in all three screens was the previously characterized sRNA FASX [17]. These results strongly suggest that a comprehensive analysis of bacterial genomes requires the combination of mathematical predictions with the collection of expression data. In the long term, testing of different conditions, especially mimicking in vivo situations by employing infection models, might lead to an increased overlap of expression detection and bioinformatics analyses.

\section{Conclusions}

We present here the identification of 55 putative sRNAs in GAS M49 by an intergenic tiling array approach. The candidate sRNA genes were expressed during growth in CDM. Forty-two of the RNAs were novel, whereas 13 RNAs have been described previously. The sequences of most of the candidates were conserved over streptococcal genomes. However, comparison of our GAS M49 sRNA expression data to another array analysis of a GAS M1 strain, and to two in silico screening methods, revealed little overlap between the different approaches. Thus, the investigation of several conditions and the combination of screening tools will be necessary to gain a comprehensive understanding of the abundance of sRNAs in GAS. The identification of novel differentially expressed sRNA genes will enhance our understanding of virulence related gene regulation in GAS. To account for specific expression patterns of putative sRNAs, infection relevant conditions combined with next generation RNA sequencing should be employed to investigate sRNA dependent regulatory networks in GAS.

\section{Methods}

Bacterial strains and culture conditions

GAS serotype M49 strain 591, a clinical isolate from a skin infection, was kindly provided by R. Lütticken (Aachen, Germany). The GAS strain was cultured in chemically defined medium (CDM) [48], Todd-Hewitt broth (Invitrogen) supplemented with $0.5 \%$ yeast extract 


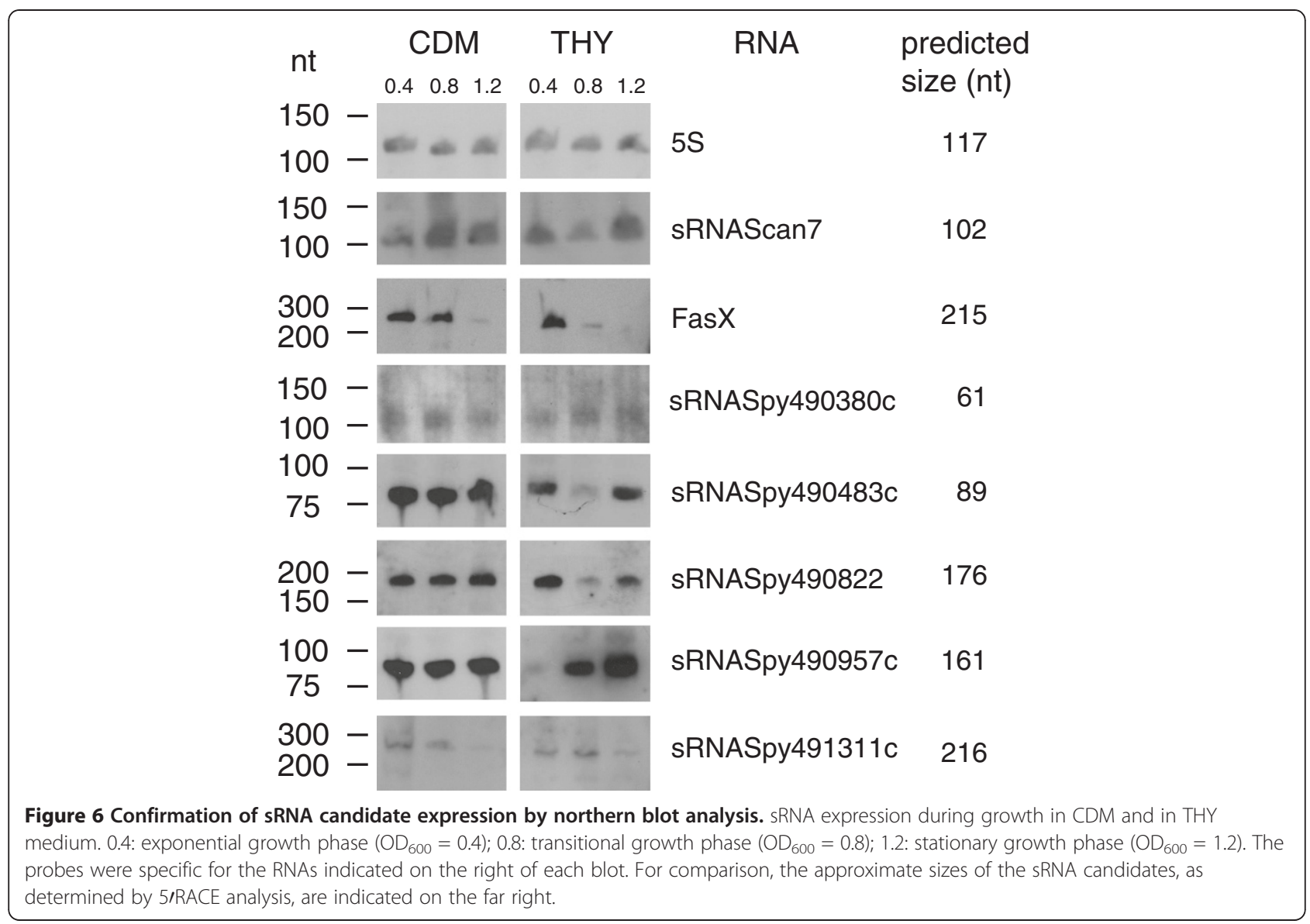

(THY; Invitrogen), or Brain-Heart-Infusion medium (BHI; Oxoid), as indicated, at $37^{\circ} \mathrm{C}$ under a $5 \% \mathrm{CO}_{2} / 20 \%$ $\mathrm{O}_{2}$ atmosphere.

\section{RNA isolation}

Total bacterial RNA from cultures grown to exponential and stationary phase of growth was isolated using the FastRNAProBlue Kit from MP Biomedicals according to manufacturer's instructions. The purified total RNA was digested with DNaseI (Ambion) to remove remaining traces of chromosomal DNA. The RNA preparation was treated with $10 \mathrm{U}$ of DNase1 for $30 \mathrm{~min}$ at $37^{\circ} \mathrm{C}$. The enzyme was subsequently heat inactivated at $72^{\circ} \mathrm{C}$ for 5 min.

\section{Enrichment of small RNAs}

Five micrograms of total RNA were fractionated using the Ambion FlashPAGE Fractionator, Ambion FlashPAGE Precast Gels, and the Ambion FlashPAGE Buffer Kit, following manufacturer's instructions. To collect the fraction of RNA molecules <200 nucleotides in length, the protocol was modified by increasing the running time from $12 \mathrm{~min}$ to up to $45 \mathrm{~min}$ at $75 \mathrm{~V}$.

\section{Labelling}

The small RNA fraction was ethanol-precipitated overnight at $-20^{\circ} \mathrm{C}$. The RNA was pelleted by centrifugation, dissolved in nuclease-free water, and labelled using the Ambion mirVana miRNA Labeling Kit following the manufacturer's instructions. In brief, this kit involves two main steps; the $3^{\prime}$ amine-modified tailing reaction, and labelling with NHS-esters. Poly(A) Polymerase and a mixture of unmodified and amine-modified nucleotides were used to add a $20-50$ nucleotide tail to the $3^{\prime}$ end of each RNA molecule in the sample. The amine-modified RNA molecules were purified and coupled to aminereactive labelled biotin moieties as NHS-esters.

\section{Design and synthesis of microfluidic microarrays}

We used a microfluidic biochip (Febit Biomed) consisting of eight independent reaction chambers, the arrays, enclosed in a cartridge for fully automated processing. Each array contains 15,625 features which are synthesized in situ inside the microchannels using the Geniom One technology (febit biomed) [49]. The 50mer probes were designed as a whole genome tiling array, covering the intergenic regions of the S. pyogenes NZ131 genome 
(NCBI accession number: NC_011375). The forward and reverse oriented probes were synthesized in separate arrays. Thus, two arrays per sample were used.

\section{Microarray hybridization and detection}

All hybridization and detection steps were carried out using a Geniom RT Analyzer (febit biomed). Hybridizations were performed overnight (16 hours) at $42^{\circ} \mathrm{C}$. Subsequently, biotin was detected with streptavidin-phycoerythrin (SAPE). A signal amplification step was added using biotinylated anti-streptavidin antibodies (Vector Laboratories) and a second incubation with SAPE (Invitrogen). Signal detection using the appropriate filter set (Cy3) of the Geniom device employed the auto-exposure function of the Geniom software. The data discussed in this publication have been deposited in the NCBI Gene Expression Omnibus [50] and are accessible through GEO Series accession number GSE31228 (http://www.ncbi.nlm.nih. gov/geo/query/acc.cgi?acc=GSE31228).

\section{Microarray data analysis}

Raw intensities were analysed and extracted using Geniom Wizard software (febit biomed) as a tab delimited text file. The data were then converted into a matrix, with rows corresponding to the features and columns corresponding to the different samples. Data analysis was performed using GeneSpring GX (version 11) software (Agilent Technologies). The array background was calculated as the median signal intensity of all "blank-control" features on the array. Data were background corrected and then normalized using quantile normalization [51]. Following normalization, a quality control step was performed that removed all data sets with a correlation coefficient less than 0.9 compared to the corresponding biological replicates. Of the original four biological replicate data sets representing cells grown in CDM, at least three were included in the analysis. The remaining probes of the biological replicates required intensity values greater than 300 on all three arrays. Regions that showed signals on probes of both strands were manually removed following the primary analysis. The statistical significance of the determined signals was tested by unpaired student's t-test with a false discovery rate of $5 \%$. Resulting data were combined with gene information from the flanking coding regions. Terminators and promoters were predicted by TransTermHP [52] (http://transterm.cbcb.umd.edu/tt/Streptococcus_pyogenes _NZ131.tt) and BDGP Neural Network Promoter Prediction [53], and BProm (www.SoftBerry.com), respectively. To investigate sRNA gene regulation, two biological replicates of growth experiments conducted in THY or BHI were included. Following data normalization, threefold signal intensity differences between various conditions were determined using the GeneSpring GX (version 11) software (Agilent Technologies). A motif search was conducted using MEME Suite [27], followed by motif analyses using TOMTOM [28], (http://meme.sdsc.edu/ meme/intro.html).

\section{5 ' RACE}

The transcriptional start sites of sRNA candidates were determined using $5^{\prime}$ RACE (Invitrogen) following the manufacturer's instructions. Briefly, first strand cDNA was synthesized using gene-specific primers (Additional file 2). The original mRNA was enzymatically removed and the $3^{\prime}$ end of the cDNA was tailed with dCTP by terminal deoxynucleotidyl transferase (TdT). PCR amplification was performed with nested, sequence-specific primers and an anchor primer provided by the $5^{\prime}$ RACE system. Primers specific for the sRNA genes tested here are listed in Additional file 2. Following amplification, PCR products were cloned into a TOPO-TA vector (Invitrogen) and sequenced (GATC Biotech AG).

\section{Quantitative reverse transcription PCR}

Acidic phenol-extracted, DNaseI-treated total RNA was reverse transcribed to generate cDNA using the FirstStrand cDNA Synthesis Kit from Invitrogen following the protocol provided by the manufacturer. For genespecific reverse transcription (RT), three reactions were performed: two strand-specific reactions with either one forward or one reverse primer, and one control reaction without any primer. Primers were designed based on the full genome sequence of S. pyogenes M49 strain NZ131 (NCBI accession number: NC011375) and are listed in Additional file 3. Three independent RT experiments were performed and all subsequent PCR reactions were performed in triplicate. Primer efficiency was tested on genomic GAS M49 DNA prior to use in RT reactions. All cDNA products were amplified by PCR with two primers specific for the respective candidate sequences. Real time PCR amplification was performed with SYBR Green (Fermentas) using an ABI PRISM 7000 Sequence Detection system (Applied Biosystems). The level of 5S RNA gene transcription was used for normalization. Relative gene expression was determined by the $\Delta \Delta \mathrm{CT}$ method [54].

\section{Northern blot analyses}

Total RNA was isolated during exponential $\left(\mathrm{OD}_{600}=0.4\right)$, transitional $\left(\mathrm{OD}_{600}=0.8\right)$, and stationary $\left(\mathrm{OD}_{600}=1.2\right)$ growth phases. RNA samples (10 $\mu \mathrm{g}$ per growth phase) were loaded onto an $8 \%$ TBE-Urea polyacrylamide gel and separated by electrophoresis. Size standards (Ultra Low Range Ladder, Fermentas) were loaded on the same gel. RNA was electroblotted onto positively charged nylon membranes (Ambion), UV cross-linked, and probed overnight with a probe complementary to a candidate sRNA. Probes were generated by PCR with the same primers as 
used for the PCR reaction in qRT-PCR experiments (listed in Additional file 3). Probes were labelled with biotin prior to hybridization (Brightstar psoralen-biotin labeling kit, Ambion). A BrightStar BioDetect Kit (Ambion) was used for detection, and autoradiography films were exposed to the luminescent blots.

\section{Additional files}

\section{Additional file 1: A: northern blot analysis of CRISPR gene expression and transcript processing in GAS M49; B: northern blot analysis of sRNASpy490957c gene expression in GAS M49. \\ Additional file 2: Sequences of primers used for 5 ' RACE. Additional file 3: Sequences of gene-specific primers employed for qRT-PCR.}

\section{Competing interests}

We are currently applying for a patent relating to the small RNAs described in this manuscript. German Patent and Trade Mark Office (DPMA), official file number: 102012104814.2.

\section{Authors' contributions}

NP participated in the design of the study, carried out experiments, analysed the microarray data, and drafted the manuscript. JN and AWK carried out experiments. VB carried out the array probe hybridisation and participated in writing the manuscript. PR performed data analyses using the MOSES bioinformatics tool. $\mathrm{AB}$ and $\mathrm{TH}^{2}$ participated in the design of the study and helped with data analysis and interpretation. JR and $\mathrm{TH}^{4}$ helped with data integration, analysis and interpretation. BK conceived of the study, participated in its design and coordination, and participated in writing the manuscript. All authors read and approved the final manuscript.

\section{Acknowledgements}

The work of NP and BK was supported by a BMBF grant in the framework of the ERA-Net PathoGenoMics 2 program (FKZ 0315437B). The work of AB and $\mathrm{TH}^{2}$ was supported by a BMBF grant ERA-NET Pathogenomics Network to the sncRNAomics project 62080061 to $\mathrm{TH}^{2}$.

\section{Author details}

${ }^{1}$ Institute of Medical Microbiology and Hospital Hygiene, University of Rostock, Schillingallee 70, 18057, Rostock, Germany. ${ }^{2}$ Institute of Medical Microbiology, Genome Research, Justus-Liebig-University, Frankfurter Strasse 107, 35392, Giessen, Germany. '3ystems Biology and Bioinformatics Group, University of Rostock, 18051, Rostock, Germany. ${ }^{4}$ Genedata AG, Margarethenstrasse 38, 4053, Basel, Switzerland. ${ }^{5}$ febit biomed GmbH, Im Neuenheimer Feld 519, 69120, Heidelberg, Germany.

Received: 4 July 2012 Accepted: 10 October 2012

Published: 13 October 2012

\section{References}

1. Brantl S: Bacterial chromosome-encoded small regulatory RNAs. Future Microbiol 2009, 4:85-103.

2. Narberhaus F, Vogel J: Regulatory RNAs in prokaryotes: here, there and everywhere. Mol Microbiol 2009, 74:261-269.

3. Waters LS, Storz G: Regulatory RNAs in bacteria. Cell 2009, 136:615-628.

4. Frohlich KS, Vogel J: Activation of gene expression by small RNA. Curr Opin Microbiol 2009, 12:674-682

5. Podkaminski D, Vogel J: Small RNAs promote mRNA stability to activate the synthesis of virulence factors. Mol Microbiol 2010, 78:1327-1331.

6. Gorke B, Vogel J: Noncoding RNA control of the making and breaking of sugars. Genes Dev 2008, 22:2914-2925.

7. Heeb S, Valverde C, Gigot-Bonnefoy C, Haas D: Role of the stress sigma factor RpoS in GacA/RsmA-controlled secondary metabolism and resistance to oxidative stress in Pseudomonas fluorescens CHAO. FEMS Microbiol Lett 2005, 243:251-258.
8. Heidrich N, Chinali A, Gerth U, Brantl S: The small untranslated RNA SR1 from the Bacillus subtilis genome is involved in the regulation of arginine catabolism. Mol Microbiol 2006, 62:520-536.

9. Gottesman S, McCullen CA, Guillier M, Vanderpool CK, Majdalani N, Benhammou J, Thompson KM, FitzGerald PC, Sowa NA, FitzGerald DJ: Small RNA regulators and the bacterial response to stress. Cold Spring Harb Symp Quant Biol 2006, 71:1-11.

10. Vanderpool CK, Gottesman S: Noncoding RNAs at the membrane. Nat Struct Mol Biol 2005, 12:285-286.

11. Papenfort K, Vogel J: Regulatory RNA in bacterial pathogens. Cell Host Microbe 2010, 8:116-127.

12. Livny J, Brencic A, Lory S, Waldor MK: Identification of 17 Pseudomonas aeruginosa sRNAs and prediction of sRNA-encoding genes in 10 diverse pathogens using the bioinformatic tool sRNAPredict2. Nucleic Acids Res 2006, 34:3484-3493.

13. Carapetis JR, Steer AC, Mulholland EK, Weber M: The global burden of group A streptococcal diseases. Lancet Infect Dis 2005, 5:685-694.

14. Cole JN, Barnett TC, Nizet V, Walker MJ: Molecular insight into invasive group A streptococcal disease. Nat Rev Microbiol 2011, 9:724-736.

15. Fiedler T, Sugareva V, Patenge N, Kreikemeyer B: Insights into Streptococcus pyogenes pathogenesis from transcriptome studies. Future Microbiol 2010, 5:1675-1694.

16. Le RA, Charpentier E: Small RNAs in streptococci. RNA Biol 2012, 9:414-426.

17. Kreikemeyer B, Boyle MD, Buttaro BA, Heinemann M, Podbielski A: Group A streptococcal growth phase-associated virulence factor regulation by a novel operon (Fas) with homologies to two-component-type regulators requires a small RNA molecule. Mol Microbiol 2001, 39:392-406.

18. Roberts SA, Scott JR: RivR and the small RNA RivX: the missing links between the CovR regulatory cascade and the Mga regulon. $\mathrm{Mol}$ Microbiol 2007, 66:1506-1522.

19. Raasch P, Schmitz U, Patenge N, Vera J, Kreikemeyer B, Wolkenhauer O: Non-coding RNA detection methods combined to improve usability, reproducibility and precision. BMC Bioinformatics 2010, 11:491.

20. Perez N, Trevino J, Liu Z, Ho SC, Babitzke P, Sumby P: A genome-wide analysis of small regulatory RNAs in the human pathogen group $A$ Streptococcus. PLoS One 2009, 4:e7668.

21. Sridhar J, Sambaturu N, Sabarinathan R, Ou HY, Deng Z, Sekar K, Rafi ZA, Rajakumar K: sRNAscanner: a computational tool for intergenic small RNA detection in bacterial genomes. PLoS One 2010, 5:e11970.

22. Gardner PP, Daub J, Tate JG, Nawrocki EP, Kolbe DL, Lindgreen S, Wilkinson AC, Finn RD, Griffiths-Jones S, Eddy SR, et al: Rfam: updates to the RNA families database. Nucleic Acids Res 2009, 37:D136-D140.

23. Tillier ER, Collins RA: The contributions of replication orientation, gene direction, and signal sequences to base-composition asymmetries in bacterial genomes. J Mol Evol 2000, 50:249-257.

24. Rocha EP: The replication-related organization of bacterial genomes. Microbiology 2004, 150:1609-1627.

25. Rutherford K, Parkhill J, Crook J, Horsnell T, Rice P, Rajandream MA, Barrell B: Artemis: sequence visualization and annotation. Bioinformatics 2000 16:944-945.

26. Facklam R: What happened to the streptococci: overview of taxonomic and nomenclature changes. Clin Microbiol Rev 2002, 15:613-630.

27. Bailey TL, Boden M, Buske FA, Frith M, Grant CE, Clementi L, Ren J, Li WW, Noble WS: MEME SUITE: tools for motif discovery and searching. Nucleic Acids Res 2009, 37:W202-W208

28. Gupta S, Stamatoyannopoulos JA, Bailey TL, Noble WS: Quantifying similarity between motifs. Genome Biol 2007, 8:R24.

29. Deltcheva E, Chylinski K, Sharma CM, Gonzales K, Chao Y, Pirzada ZA, Eckert MR, Vogel J, Charpentier E: CRISPR RNA maturation by trans-encoded small RNA and host factor RNase III. Nature 2011, 471:602-607.

30. Storz G, Vogel J, Wassarman KM: Regulation by small RNAs in bacteria: expanding frontiers. Mol Cell 2011, 43:880-891.

31. Thomason MK, Storz G: Bacterial antisense RNAs: how many are there, and what are they doing? Annu Rev Genet 2010, 44:167-188.

32. Gottesman S, Storz G: Bacterial small RNA regulators: versatile roles and rapidly evolving variations. Cold Spring Harb Perspect Biol 2011, 3. doi:10.1101/cshperspect.a003798.

33. Ramirez-Pena E, Trevino J, Liu Z, Perez N, Sumby P: The group A Streptococcus small regulatory RNA FasX enhances streptokinase activity 
by increasing the stability of the ska mRNA transcript. Mol Microbiol 2010, 78:1332-1347.

34. Mangold M, Siller M, Roppenser B, Vlaminckx BJ, Penfound TA, Klein R, Novak R, Novick RP, Charpentier E: Synthesis of group A streptococcal virulence factors is controlled by a regulatory RNA molecule. $\mathrm{Mol}$ Microbiol 2004, 53:1515-1527.

35. Biswas I, Germon P, McDade K, Scott JR: Generation and surface localization of intact M protein in Streptococcus pyogenes are dependent on sagA. Infect Immun 2001, 69:7029-7038.

36. Betschel SD, Borgia SM, Barg NL, Low DE, De Azavedo JC: Reduced virulence of group A streptococcal Tn916 mutants that do not produce streptolysin S. Infect Immun 1998, 66:1671-1679.

37. Trevino J, Perez N, Sumby P: The $4.5 S$ RNA component of the signal recognition particle is required for group A Streptococcus virulence. Microbiology 2010, 156:1342-1350.

38. Beaume M, Hernandez D, Docquier M, Delucinge-Vivier C, Descombes $P$, Francois $P$ : Orientation and expression of methicillin-resistant Staphylococcus aureus small RNAs by direct multiplexed measurements using the nCounter of NanoString technology. J Microbiol Methods 2011, 84:327-334.

39. Chen Y, Indurthi DC, Jones SW, Papoutsakis ET: Small RNAs in the genus Clostridium. MBio 2011, 2:e00340-10.

40. Kumar R, Shah P, Swiatlo E, Burgess SC, Lawrence ML, Nanduri B: Identification of novel non-coding small RNAs from Streptococcus pneumoniae TIGR4 using high-resolution genome tiling arrays. BMC Genomics 2010, 11:350.

41. Mraheil MA, Billion A, Mohamed W, Mukherjee K, Kuenne C, Pischimarov J, Krawitz C, Retey J, Hartsch T, Chakraborty T, et al: The intracellular sRNA transcriptome of Listeria monocytogenes during growth in macrophages. Nucleic Acids Res 2011, 39:4235-4248.

42. Tsui HC, Mukherjee D, Ray VA, Sham LT, Feig AL, Winkler ME: Identification and characterization of noncoding small RNAs in Streptococcus pneumoniae serotype 2 strain D39. J Bacteriol 2010, 192:264-279.

43. Marraffini LA, Sontheimer EJ: Self versus non-self discrimination during CRISPR RNA-directed immunity. Nature 2010, 463:568-571.

44. Haft DH, Selengut J, Mongodin EF, Nelson KE: A guild of 45 CRISPRassociated (Cas) protein families and multiple CRISPR/Cas subtypes exist in prokaryotic genomes. PLoS Comput Biol 2005, 1:e60.

45. Livny J, Fogel MA, Davis BM, Waldor MK: sRNAPredict: an integrative computational approach to identify sRNAs in bacterial genomes. Nucleic Acids Res 2005, 33:4096-4105.

46. Livny J, Waldor MK: Identification of small RNAs in diverse bacterial species. Curr Opin Microbiol 2007, 10:96-101.

47. Arnvig KB, Young DB: Identification of small RNAs in Mycobacterium tuberculosis. Mol Microbiol 2009, 73:397-408.

48. van de Rijn I, Kessler RE: Growth characteristics of group A streptococci in a new chemically defined medium. Infect Immun 1980, 27:444-448.

49. Baum M, Bielau S, Rittner N, Schmid K, Eggelbusch K, Dahms M, Schlauersbach A, Tahedl H, Beier M, Guimil R, et al: Validation of a novel, fully integrated and flexible microarray benchtop facility for gene expression profiling. Nucleic Acids Res 2003, 31:e151.

50. Edgar R, Domrachev M, Lash AE: Gene Expression Omnibus: NCBI gene expression and hybridization array data repository. Nucleic Acids Res 2002, 30:207-210.

51. Bolstad BM, Irizarry RA, Astrand M, Speed TP: A comparison of normalization methods for high density oligonucleotide array data based on variance and bias. Bioinformatics 2003, 19:185-193.

52. Kingsford CL, Ayanbule K, Salzberg SL: Rapid, accurate, computationa discovery of Rho-independent transcription terminators illuminates their relationship to DNA uptake. Genome Biol 2007, 8:R22.

53. Reese MG: Application of a time-delay neural network to promoter annotation in the Drosophila melanogaster genome. Comput Chem 2001, 26:51-56.

54. Livak KJ, Schmittgen TD: Analysis of relative gene expression data using real-time quantitative PCR and the 2(-Delta Delta C(T)) Method. Methods 2001, 25:402-408.

doi:10.1186/1471-2164-13-550

Cite this article as: Patenge et al:: Identification of novel growth phaseand media-dependent small non-coding RNAs in Streptococcus pyogenes M49 using intergenic tiling arrays. BMC Genomics 2012 13:550.

\section{Submit your next manuscript to BioMed Central and take full advantage of:}

- Convenient online submission

- Thorough peer review

- No space constraints or color figure charges

- Immediate publication on acceptance

- Inclusion in PubMed, CAS, Scopus and Google Scholar

- Research which is freely available for redistribution 